\title{
THE TOTAL SPACE-TIME OF A POINT CHARGE AND ITS CONSEQUENCES FOR BLACK HOLES
}

\author{
LEONARD S. ABRAMS
}

\begin{abstract}
Singularities associated with an incomplete space-time $S$ are not uniquely defined until a boundary $B$ is attached to it. [The resulting space-time-with-boundary, $\bar{S} \equiv S \cup B$, will be termed a "total" space-time (TST).] Since an incomplete space-time is compatible with a variety of boundaries, it follows that $\mathrm{S}$ does not represent a unique universe, but instead corresponds to a family of universes, one for each of the distinct TSTs. It is shown here that the boundary attached to the Reissner-Nordström space-time for a point charge is invalid for $q^{2}<m^{2}$. When the correct boundary is used, the resulting TST is inextendible. This implies that the Graves-Brill black hole cannot be produced by gravitational collapse. The same is true of the Kruskal-Fronsdal black hole for the point mass, and for those black holes which reduce to the latter for special values of their parameters.
\end{abstract}

\section{INTRODUCTION}

As is well known [1], the singularity structure of a casually incomplete, not necessarily maximal space-time $S=(M, g)$ is not uniquely specified by $(M, g)$ alone, but requires in addition that a boundary $B$ be attached thereto [2].1] [The resulting object, $T \equiv S \cup B$, will be termed a 'total' space-time (TST).] Since an incomplete space-time is compatible with a variety of boundaries (Geroch, 1968, p. 451) [3], it follows that $S$ by itself cannot represent a unique universe - instead, it corresponds to a family of universes, one for each of its topologically distinct boundaries.

Recognition of this fact immediately gives rise to the question: "Which of the possible boundaries of $S$ should be attached to it?". The answer depends on how much additional information is available; if nothing more is known than $(M, g)$ itself, then one is free to attach any of its mathematically admissible boundaries, the only remaining problem being the physical interpretation of the result. If, on the other hand, one also knows the universe $U$ from which $S$ was derived, or which the TST of $S$ is intended to represent, then there is no freedom whatever to choose $B$ - the boundary is uniquely determined by $U$, just as are such things as the symmetries of $S$, whether $S$ is static, etc. In some cases this determination could be quite difficult, while

\footnotetext{
Key words and phrases. Incomplete space-times; Boundaries; Black holes.

Published in Int. J. Theor. Phys. 35 (1996) pp. 2661-2677.

${ }^{1}$ For simplicity, whenever an order of differentiability is required to completely specify a concept (e.g., manifold, extension, equivalent, etc), "analytic" is always to be understood.
} 
in others it could be trivial. An example of the latter situation would be a $U$ consisting of a single point source, for then the boundary of the TST of $S$ is evidently a line through the location of the source.

Incidentally, note that since a TST is a space-time-with-boundary, the criteria for equivalence and extendibility of TST are necessarily different from those applicable to space-times. Specifically, equivalence of TSTs requires not only that their $S$ components be isometric $\| 4]$, but also that their $B$ components be homeomorphic. Likewise, extendibility of a TST requires not only extendibility of its $S$ component, but also that its $B$ component be preserved - i.e., that the image of $B$ be homeomorphic to $B$ itself.

Admittedly, in most cases the only difference between the TSTs associated with different choices of a boundary for a given $S$ is the topology of the singularities, as is the case in the example in Hawking and Ellis [2]. However, when $S$ is extendible, the choice of boundary to be attached to it may well affect the very existence of a singularity of the resulting TST. For example, consider the following two-dimensional, Riemannian case: $(M, g)=\left(D_{1}, g_{E}\right)$, where $D_{1}$ denotes the interior of the unit disk in $R^{2}$ (i.e., $x^{2}+y^{2}<1$ ), and $g_{E}$ denotes the Euclidean metric: $d x^{2}+d y^{2}$. If the boundary is taken to be $S^{1}$, then there is no singularity at the boundary and the space possesses an extension to all of $R^{2}$, namely $\left(R^{2}, g_{E}\right)$. On the other hand, if the boundary is taken to be a point (e.g., by identifying all the points of the circle $x^{2}+y^{2}=1$ ), then there is a quasiregular singularity at the boundary and no extension is possible.

As is well known, the Reissner-Nordström space-time $S_{R N}$ is timelike incomplete [5], so that by the argument in the first paragraph it cannot represent a unique universe. Moreover, examination of Nordström's derivation shows that it involves a tacit assumption which is invalid for $q^{2}<m^{2}$ and which attached a boundary to $S_{R N}$ that is incompatible with the pointcharge universe from which $S_{R N}$ was derived. As a result, the RN TST does not represent a point charge for $q^{2}<m^{2}$, and a fortiori, neither does its black-hole-containing extension [6]. When the space-time for a point charge is correctly derived and the appropriate boundary is attached, the resulting TST is inextendible and devoid of a black hole. Since this TST is necessarily the limit of the space-time of any spherically symmetric, nonrotating, charged star that is collapsing to a point, it follows that such collapse cannot produce a black hole.

Since there is no way of deriving the Graves-Brill (GB) space-time from the set of postulates characterizing a point charge, nor of producing it by gravitational collapse, it follows that it has neither a theoretical nor a physical justification for its existence. Thus it, and the black hole which it contains, are simply artifacts of a historical error. The same is true of the point-mass black hole [7], [8], since it is based on Hilbert's space-time for

\footnotetext{
${ }^{2}$ As can be seen from p. 26 of this reference, equivalence also requires that the isometry be orientation- and time-orientation-preserving; hence any reference herein to an isometry will be understood to mean one having these properties.
} 
a point mass, whose derivation (in 1917) involved the same erroneous assumption [9] as was used by Nordström.

The purpose of this paper is to derive the correct TST for a point charge and to show how the above-mentioned consequences come about.

The plan of the paper is as follows: Section 2 is devoted to showing that Nordström's [10] derivation made use of an unjustified assumption, which among other things resulted in the attachment of a boundary that is incompatible with the point-charge universe when $q^{2}<m^{2}$. Section 3 lists the historical postulates on which Reissner's [11] and Nordström's derivations were based. Section 4 contains a derivation of the point-charge space-time for $q^{2}<m^{2}$ which does not make use of Nordström's assumption, and in the process proves that the assumption is invalid for the case in question. In addition, it also contains a proof that the historical postulates do not give rise to a unique space-time, but to a one-parameter family of inequivalent space-times, and thus that these postulates must be supplemented by one which determines the limiting value of a certain invariant as the point charge is approached. Section 5 discusses the physical significance of the supplemental hypothesis, and proposes a criterion for choosing among the infinitely many possible values of the invariant. In Section 6 the metric which results from this choice is obtained, and the associated TST is shown to be equivalent to that found by Pekeris [12]. Section 7 deals with the phenomenon of a nonspinning, spherically symmetric, charged star undergoing gravitational collapse to a point. It is shown that such collapse can never produce a GB black hole. Section 8 shows that because of the infinite red shift at the horizon of the GB space-time, there are in principle no phenomena explainable by a GB black hole that would not be equally well explained by Pekeris' TST, so that the former is unnecessary from a phenomenological standpoint (and considerably more complicated than Pekeris' TST). Finally, the principal conclusions of the paper are summarized in Section 9, together with a brief discussion.

\section{HISTORICAL BACKGROUND}

The first attempt to determine the space-time of a point charge was that of Reissner 111], However, his derivation was only valid for the case $q^{2}>$ $m^{2}$, where $m$ denotes the particle's field-producing mass (i.e., that which produces Kepler-like orbits far from the point charge) and $q$ denotes its charge (in relativistic esu) In addition, it contained an unknown constant $\left[h\left(0^{+}\right)\right.$in Reissner's notation] whose value was tacitly assumed.

A year or so later, a derivation applicable to all values of $q^{2} / m^{2}$ was presented by Nordström [10]. Taking the point charge to be at $x=y=z=$

\footnotetext{
${ }^{3}$ In the referenced paper, I asserted $(\S 6)$ that since Schwarzschild's and Hilbert's spacetimes had different singularity structures, it followed that the two space-times were inequivalent (cf. Sachs and Wu [4], § 1.3). This is incorrect - as shown here, it is only their TSTs which are inequivalent.
} 
0 , the starting point of his derivation was the static, spherically symmetric metric

$$
g_{s s s}(r \mid 0) \equiv A(r) d t^{2}-B(r) d r^{2}-C(r) d \Omega^{2}
$$

where

$$
r \equiv\left(x^{2}+y^{2}+z^{2}\right)^{1 / 2}
$$

and

$$
d \Omega^{2} \equiv d \theta^{2}+d \phi^{2} \sin ^{2} \theta
$$

[The $r \mid 0$ in the argument of $g_{\text {sss }}$ signifies that in terms of $r$, the position of the point charge is described by $r=0$. A similar notation will be used for subsequent metrics.]

Substituting equation (1) into the variational equivalent of the EinsteinMaxwell field equations gave Nordström three ordinary differential equations (ODE) for the three unknown coefficients. Instead of solving these (which is done here in Appendix A), Nordström argued that one could always introduce a new radial variable, $r^{*}$ say, via

$$
r^{*}=[C(r)]^{1 / 2}
$$

This transformation carries equation (1) into

$$
g_{s s s}^{*}\left(r^{*} \mid r_{0}^{*}\right)=A^{*}\left(r^{*}\right) d t^{2}-B^{*}\left(r^{*}\right) d r^{* 2}-r^{* 2} d \Omega^{2},
$$

and, as follows directly from equation (4), assigns the value

$$
r^{*}=r_{0}^{*}=[C(0+)]^{1 / 2}
$$

to the location of the point charge. Setting $C^{*}=r^{* 2}$ into the abovementioned ODE, Nordström then solved for $A^{*}$ and $B^{*}$, obtaining expressions for these quantities which contained but one constant of integration, $\alpha_{N}$. This constant was readily shown to be equal to $2 m$, giving the results

$$
\begin{gathered}
A^{*}\left(r^{*}\right)=A_{N}\left(r^{*}\right) \equiv 1-2 m / r^{*}+q^{2} / r^{* 2} \\
B^{*}\left(r^{*}\right)=B_{N}\left(r^{*}\right) \equiv 1 / A_{N}\left(r^{*}\right)
\end{gathered}
$$

However, the fact that $C(r)$ is unknown at the stage where $r^{*}$ is introduced shows that the value of $r_{0}^{*}$ cannot be determined from equation (田). Moreover, the fact that $r=0$ is the location of the point charge and thus a singularity of the field makes it impossible to appeal to 'elementary flatness' to conclude that $C(0+)=0$. Consequently, Nordström's solution seemingly involves an unknown and unknowable constant, namely the $r^{*}$ coordinate of the point charge. [This would appear, for example, in the expression for the proper distance from the point charge to an event having $r^{*}=r_{P}^{*}$, namely

$$
d\left(r_{P}^{*}\right)=\int_{r_{0}^{*}}^{r_{P}^{*}}\left[B^{*}\left(r^{*}\right)\right]^{1 / 2} d r^{*}
$$


or in that for the proper volume from the point charge to $r_{P}^{*}$, etc.] However Nordström tacitly assumed that $r^{*}=\left(x^{2}+y^{2}+z^{2}\right)^{1 / 2}$, which implies that the location $(x=y=z=0)$ of the point charge in terms of $r^{*}$ is given by $r^{*}=0$. But for $q^{2}<m^{2}, B_{N}^{*}$ tends to $\infty$ as $r^{*} \downarrow r_{+}^{*} \equiv m+\left(m^{2}-q^{2}\right)^{1 / 2}$, so for this case Nordström's metric is only defined for $r^{*}>r_{+}^{*}$. Moreover, this assumption also results in the attachment of a particular boundary to this space-time: a 2-sphere at $r^{*}=r_{+}^{*}$ in the sections $t=$ const.

Although Nordström only claimed that his space-time represented a point charge for $q^{2}>m^{2}$, this was lost sight of in the ensuing years, and $S_{R N}$ has come to be regarded as the space-time of a point charge for all values of $q^{2} / m^{2}$. Following historical usage, the metric obtained from equation (5) by setting $A^{*}=A_{N}, B^{*}=B_{N}$, and assuming that $r^{*}=\left(x^{2}+y^{2}+z^{2}\right)^{1 / 2}$ will henceforth be termed the Reissner-Nordström metric and will be denoted by $g_{R N}\left(r^{*} \mid 0\right)$ :

$$
g_{R N}\left(r^{*} \mid 0\right)=A_{N}\left(r^{*}\right) d t^{2}-B_{N}\left(r^{*}\right) d r^{* 2}-r^{* 2} d \Omega^{2}
$$

Subsequent derivations of the point-charge metric were carried out by Hönl and Papapetrou [13] and Pekeris [12]. In both cases the authors applied the tecnique used by Schwarzschild [14] in his derivation of the pointmass metric, thereby avoiding Nordström's error; but in each case ad hoc assumptions were used to evaluate one of the integration constants and thereby obtain a unique field.

Analytic extensions of the RN space-time were subsequently obtained by Graves and Brill [6] for $q^{2}<m^{2}$ and by Carter [5] for $q^{2}=m^{2}$.

Finally, the point of view adopted here is the generally accepted one that charge of either sign increases the field-producing mass, and that charge without matter is impossible; the first of these implies that $q^{2} \leq m^{2}$, while the second reduces the latter to $q^{2}<m^{2}$. (This implies that point-charge space-times for which $q^{2} \geq m^{2}$ are nonexistent, and thus so, too, is the Carter black hole.) This inequality is to be understood to hold in the following sections.

\section{THE HISTORICAL POSTULATES}

The postulates that until now have been regarded as characterizing the point-charge space-time $S_{P C} \equiv\left(M_{P C}, g_{P C}\right)$ may be gleaned from Reissner's paper. In vernacular terms, they require that:

\footnotetext{
${ }^{4}$ In 10$]$, just under equation (5), he states, "..where $e\left(r^{*}\right)$ denotes the total charge in a sphere of radius $r^{*}$." And just above equation (19a), he states, "Because of the spherical symmetry we have, of course for the components of $U$ in the directions of the axes of coordinates in space

$$
U_{\tau}=x_{\tau} c \alpha /\left(r^{* 3}\right) \quad \tau=1,2,3^{\prime \prime}
$$

having previously defined $U=c \alpha /\left(r^{* 2}\right)$ in equation (19). [Note: $r$ in Nordström's paper has been changed to $r^{*}$ here so as to conform to the notation adopted in the present paper.] Neither of these statements makes sense unless $r^{*}$ is regarded as $\left(x^{2}+y^{2}+z^{2}\right)^{1 / 2}$.
} 
(i) $M_{P C}$ consist of $R^{4}$ less a line through the point charge $\left(\equiv M_{0}\right)$.

That $g_{P C}$ be:

(ii) Of Lorentz signature.

(iii) Analytic.

(iv) Static.

(v) Spherically symmetric about the point charge.

(vi) A solution of the Einstein-Maxwell field equations.

(vii) Flat at spatial infinity (i.e., $g_{i j} \rightarrow \eta_{i j}$ ).

And that:

(viii) $S_{P C}$ be inextendible.

\section{THE EXTENT TO WHICH THE HISTORICAL POSTULATES DETERMINE THE METRIC}

Let $K$ denote $R^{4}$ considered as the analytic manifold arising from the single-chart atlas $\left(R^{4}, I d\right)$, and $(t, x, y, z)$ the natural coordinates (O'Neill, 1983, p. 1) [15] thereon. Without loss of generality we shall take the charge to be located at $x=y=z=0$. Let $L$ denote the line $x=y=z=0$, so that $M_{0}=K \backslash L$.

As is well known [16], a metric satisfying (ii)-(v) everywhere on $M_{0}$ can always be expressed in the form

$$
\begin{array}{r}
g_{P C}(x, y, z \mid 0,0,0)=A(r) d t^{2}-F(r)\left(d x^{2}+d y^{2}+d z^{2}\right) \\
-G(r)(x d x+y d y+z d z)^{2}
\end{array}
$$

where

$$
r=\left(x^{2}+y^{2}+z^{2}\right)^{1 / 2}
$$

and $A, F, G$ are analytic functions of $r$, satisfying

$$
A, F, G>0
$$

so as to ensure compliance with (ii).

Transforming to quasipolar coordinates related to $x, y, z$ in the customary way, we find that equation (11) becomes (with a slight abuse of notation)

$$
g_{P C}(r \mid 0)=A(r) d t^{2}-B(r) d r^{2}-C(r) d \Omega^{2}
$$

while condition (13) becomes

$$
A, B, C>0
$$

Substituting equation (14) into the Einstein-Maxwell equations, solving for $A, B$, and $C$, and imposing (vii) gives (see Appendix A)

$$
\begin{gathered}
A(r)=1-2 m / C^{1 / 2}+q^{2} / C \\
B(r)=C^{\prime 2} /(4 A C)
\end{gathered}
$$

where the prime denotes differentiation with respect to $r$. These expressions coincide with those found by Stavroulakis [17] for the exterior of a static, 
spherically symmetric charged body when the cosmological constant in his result is set to zero, and with $q=0$, to those obtained for the point mass in Oliver [18] (this paper was the first in which the point-mass field equations were integrated without eliminating one of the unknowns).

However, as shown in Appendix A, for a point charge, $C$ must also be an analytic function of $r$ such that

$$
\begin{array}{lll}
C(r)>0 & \text { for } & r>0 \\
C^{\prime}(r)>0 & \text { for } & r>0 \\
C / r^{2} \rightarrow 1 & \text { as } & r \rightarrow \infty
\end{array}
$$

All that is necessary in order to comply with the remaining historical postulates is to impose whatever further conditions are required to ensure that $A$ is positive [it is also necessary that $B$ be positive on $M_{0}$, but conditions (17)-(19) show that this is assured if $A>0$ for $r>0$ ] and that (viii) is satisfied.

As to the first of these, Appendix B shows that $A$ will be positive iff

$$
\sqrt{C}>r_{+}^{*} \equiv m+\left(m^{2}-q^{2}\right)^{1 / 2}
$$

and since this must hold for all $r>0$, it follows that

$$
b \equiv[C(0+)]^{1 / 2} \geq r_{+}^{*}>0
$$

Since this condition is necessary to ensure the positivity of $A$, it follows that Nordström's assumption, which requires that $b=0$, is invalid.

As to the second, the fact that the metric is analytic everywhere on $M_{0} \equiv$ $K \backslash L$ shows that (viii) will be satisfied only if $L$ is singular. Now, Appendix $\mathrm{C}$ shows that the Kretschmann scalar $f=R_{i j k m} R^{i j k m}$ tends to infinity as $C \rightarrow 0$ and is bounded otherwise. In view of equations (19) and (22), this means that there are no scalar curvature singularities on $L$. But as is well known, it is possible to have a singularity without any curvaturerelated scalar being unbounded [a so-called quasiregular singularity (Ellis and Schmidt, 1977, p. 944) [19]]. To explore this possibility, let us first observe that the boundary $r=0$ must be a point in each spatial section, so as to comply with the fact that the associated TST is intended to represent a universe consisting of a single point source. Next, suppose that $b \geq r_{+}^{*}$, and consider the geodesic circle $\gamma: t=t_{0}, \theta=\pi / 2, r=\epsilon$. Its proper circumference clearly tends to $2 \pi b>0$ [from equation (14)] as $\epsilon \downarrow 0$, while its proper radius is easily seen to tend to zero, so that the ratio of the former to the latter tends to $\infty$. This shows that $r=0$ is a singularity, since such behavior makes it impossible to map an infinitesimal neighborhood of $r=0$ into an interior portion of some larger space-time. (Note that the image of $r=0$ under any such map must be a point in each spatial section, since otherwise the boundary attached to $M_{0}$ would not be homeomorphic to its image in the map, as is required for extendibility of a TST.) Consequently, postulate (viii) is satisfied for any value of $b \geq r_{+}^{*}$. [Despite appearances, the 
foregoing argument is coordinate independent, since equation (14) is unique up to transformations of the form $t=k \bar{t}+p, r=h(\bar{r})$, and neither of these alters the proper radius or proper circumference of $\gamma$.] It is the presence of this singularity which makes the TST inextendible.

Moreover, just as in the case of the point mass [9], so here, too, the value of $C[P]$ at any event $P$ is a scalar invariant of $g_{P C}(r \mid 0)$, and thus the same is true of its limit $\left(b^{2}\right)$ as $P$ approaches the point charge. Consequently, space-times whose metrics are of the form of $S_{P C}$ with distinct values of $b$ are inequivalent. Hence we conclude:

- The historical postulates do not lead to a unique space-time, but to a one-parameter family of inextendible, inequivalent space-times. In order to obtain a unique field, it is necessary to supplement those postulates by one which fixes the value of $C(0+)$.

\section{THE SUPPLEMENTAL POSTULATE}

We have seen in the previous section that in order to arrive at a unique field, it is necessary to supplement the historical postulates for the point charge by one which fixes the value of $b=[C(0+)]^{1 / 2}$. Now, as shown in Appendix D, the limiting acceleration of a neutral test particle approaching the point charge on a radial geodesic of equation (14), as measured by a sequence of fixed observers, is given by

$$
a_{0}=\frac{\left|b m-q^{2}\right|}{b^{2} \sqrt{b^{2}-2 b m+q^{2}}} \equiv \frac{\left|b m-q^{2}\right|}{b^{2} \sqrt{(b-m)^{2}+q^{2}-m^{2}}}
$$

From this it is evident that fixing the limiting value of the locally measured acceleration of a radially approaching neutral test particle is equivalent to fixing $b$. For the same reason as stated in connection with the point mass [9], we shall take this limiting acceleration to be the same $(\infty)$ as in the Newtonian case, thereby supplementing the historical postulates with the following:

(ix) The limiting value of the locally measured acceleration of a neutral test particle approaching the point charge on a radial geodesic is infinite.

\section{THE RESULTING METRIC}

Clearly, the only value of $b$ consistent with equation (22) which makes the RHS of equation (23) infinite is

$$
b=r_{+}^{*}=m+\left(m^{2}-q^{2}\right)^{1 / 2}
$$

The simplest $C$ satisfying the earlier requirements as well as equation (24) is given by

$$
C_{P} \equiv\left(r+r_{+}^{*}\right)^{2}
$$


which reduces equations (16) and (17) to respectively

$$
\begin{gathered}
A_{P}(r)=1-2 m /\left(r+r_{+}^{*}\right)+q^{2} /\left(r+r_{+}^{*}\right)^{2} \\
B_{P}(r)=\left[A_{P}(r)\right]^{-1}
\end{gathered}
$$

It is readily verified via the transformation $r+r_{+}^{*}=\left[\bar{r}^{3}+\left(r_{+}^{*}\right)^{3}\right]^{1 / 3}$ that the resulting space-time is isometric to that of Pekeris, and since the boundary atttached to the latter is likewise pointlike in any spatial section, the two TSTs are equivalent. Moreover, since Nordström's and the just-derived version of Pekeris' space-times are isometric via the transformation $r^{*}=$ $r+r_{+}^{*}$, it follows that the actual location of the point charge in terms of $r^{*}$ is given by $r^{*}=r_{+}^{*}$, so that the 2 -sphere boundary resulting from Nordström's assumption coincides with the position of the point charge, which is clearly incompatible with the pointlike nature of the source.

\section{GRAVITATIONAL COLLAPSE}

Eiesland [16] showed that any spherically symmetric solution of the Einstein-Maxwell field equations is static. In particular, then, this last must be true of the 'exterior' metric of a charged, nonrotating, nonradiating spherically symmetric object ('star') undergoing gravitational collapse. Consequently, this metric must satisfy the same postulates as for the point charge, namely (viii) and (ix). As can be seen from Appendix A, satisfaction of postulates (ii)-(vii) when applied to a metric of the form of equation (14) entails that $A$ and $B$ be given by equations (16) and (17), respectively, and together with condition (15), that $C$ be a positive, analytic, strictly monotonic increasing function of $r$ over the $r$ range for which the exterior metric is valid - i.e., for $r>r_{b}(t)$, where $r_{b}(t)$ denotes the $r$ coordinate of the star's boundary at time $t$.

Since the set of $C$ 's which, together with equations (16) and(17), give rise to a metric satisfying (ii)-(vii) for $r>r_{b}(t)$ may obviously be larger than that satisfying (ii)-(ix) for $r>0$, it cannot be asserted that the exterior metric is the 'same' as that of the point charge (in the sense of having the same values of $C$ at the same proper distances from the center of symmetry). However, if the star is sufficiently massive as to collapse to a point, then as it does so, the difference between the actual exterior metric and the metric of the point charge must vanish as $r \downarrow 0$. Consequently, in that limit the exterior metric becomes that of the point charge. Since the point-charge space-time whose metric was obtained in the previous section has no event horizon, it follows that a GB black hole cannot be formed during the collapse of a charged star. Thus, the correction of Nordström's error not only eliminates the point charge as a possible source of such black holes, but simultaneously deprives the latter of the only mechanism that has been proposed for their production. 


\section{THE GB BLACK HOLE IS UNNECESSARY}

Just as in the case for the point mass, so here, too, there is an infinite red shift in the GB space-time as $r^{*} \downarrow r_{+}^{*}$. Because of this, all that an outside observer can ever know of phenomena involving the GB black hole must arise from information originating outside the hole - i.e., from the RN space-time. But the latter is diffeomorphic to that of Pekeris - that is to say, everything that takes place outside the hole would occur in the identical fashion if the GB space-time were replaced by Pekeris'; it is impossible to determine which space-time is "really" present without going to the boundary. Thus, any observations that could be explained by postulating the presence of a GB black hole would be equally explained by postulating the existence of a Pekeris 'point' charge at the boundary of the black hole. Consequently, there is no need to invoke a GB black hole to explain any set of observations. Pekeris' black 'point' will do an equally effective job, and its topology is far simpler.

\section{CONCLUSIONS}

1. Nordström's assumptions that the radial coordinate in his metric is related to $x, y, z$ via $r^{*}=\left(x^{2}+y^{2}+z^{2}\right)^{1 / 2}$ is not only invalid, but also leads to the attachment of a boundary to his space-time that is incompatible with the pointlike nature of the source.

2. The historical postulates restrict the manifestly static and spherically symmetric form of the point-charge metric to equation (14) cum equations (16)-(20), where (with $b \equiv[C(0+)]^{1 / 2}$ )

$$
b \geq r_{+}^{*}=m+\left(m^{2}-q^{2}\right)^{1 / 2}
$$

Thus, those postulates give rise to a one-parameter family of inequivalent space-times; to obtain a unique field, they must be supplemented by one which fixes the value of $b$. Since the minimum value of $b$ is necessarily $\geq r_{+}^{*}>0$ in order to ensure the Lorentz signature of the metric, it follows that however this supplemental postulate is chosen, Nordström's assumption, which requires that $b=0$, is invalid.

3 . The limiting value $a_{0}$ of the locally measured acceleration of a neutral test particle approaching the point charge on a radial geodesic fixes $b$. The supplemental postulate therefore requires a choice of $a_{0}$, which on the basis of the Newtonian analogy is here taken to be infinite.

4. The point-charge metric resulting from this choice is

$$
\begin{array}{cc}
g_{P}=\left[1-2 m /\left(r+r_{+}^{*}\right)+q^{2} /\left(r+r_{+}^{*}\right)^{2}\right] d t^{2} & \\
-\left[1-2 m /\left(r+r_{+}^{*}\right)+q^{2} /\left(r+r_{+}^{*}\right)^{2}\right]^{-1} d r^{2} & \\
-\left(r+r_{+}^{*}\right)^{2} d \Omega^{2} & \text { for } r>0
\end{array}
$$

whose associated TST is equivalent to Pekeris'.

5. The 'exterior' metric for a charged, spherically symmetric star undergoing gravitational collapse is of the same form as that for a point charge, 
and as the collapse proceeds must approach $g_{P}$. Thus no black hole can be formed by such collapse.

6. Any observations that would be consistent with the existence of a GB black hole would be equally consistent with a Pekeris black 'point' and thus GB black holes are unnecessary to explain any phenomena.

7. Possessing neither a theoretical justification (i.e., a mathematical chain from a set of postulates characterizing a particular universe to the GB black hole) nor a mechanism for its production, the GB black hole is nothing more than an artifact of a historical error.

8. Conclusion 7 applies equally well to the Kruskal-Fronsdal black hole, since by setting $q=0$ in Nordström's TST reduces it to that erroneously derived by Hilbert for the point mass [9] and reduces the GB space-time to that obtained by Kruskal [ [8] and Fronsdal [7]. It also applies [20] to the black hole found by Lake and Roeder [21] for the point mass when $\Lambda \neq 0$.

It should be noted that what has been termed Pekeris' black 'point' does not have all the properties of a point in Euclidean 3-space - in particular, although the proper radius and proper volume of a sphere about $r=0$ passing through $r=\epsilon$ go to zero as $\epsilon \downarrow 0$, the proper circumference and proper area of the sphere approach nonzero values. Were it to have all the properties of an ordinary point, of course, it would not be a singularity of the TST, as is required of a point source.

For $q=0$, the TST obtained here was also found by Janis et al. [22] on the basis of entirely different considerations.

\section{ApPENDIX A. THE MOST GENERAL SOLUTION SATISFYING THE HISTORICAL POSTULATES}

Making the obvious modifications in the equations [Tolman, (1934) p. 259, equation (102.6)] [23] satisfied by the electromagnetic field tensor $F_{i j}$ in empty space, necessitated by the use of $C$ rather than $r^{2}$ in equation (1), we find as usual that all $F^{i j}$ vanish except for $F^{41}=-F^{14}$, and that the former satisfies (a prime denotes differentiation with respect to $r$ )

$$
\left(\ln F^{41}\right)^{\prime}+[\ln (C \sqrt{A B})]^{\prime}=0
$$

whence

$$
\phi^{\prime}=-A B F^{41}=-K \sqrt{A B} / C
$$

where $\phi$ denotes the 'time' component of the generalized potential and $K$ is an integration constant. Substituting these results into the expression [Tolman, p. 261, equation (104.1)] [23] for Maxwell's energy-momentum tensor, we obtain

$$
T_{1}^{1}=-T_{2}^{2}=-T_{3}^{3}=T_{4}^{4}=K^{2} /\left(2 C^{2}\right)
$$

whence the nonvanishing and independent field equations read (cf. [9])

$$
-8 \pi T_{1}^{1}=-1 / C+C^{\prime 2} /\left(4 B C^{2}\right)+A^{\prime} C^{\prime} /(2 A B C)=-f_{E}
$$




$$
\begin{gathered}
\text { (A.5) }-8 \pi T_{2}^{2}=\begin{array}{c}
C^{\prime \prime} /(2 B C)+A^{\prime \prime} /(2 A B)-C^{\prime 2} /\left(4 B C^{2}\right) \\
-B^{\prime} C^{\prime} /\left(4 B^{2} C\right)-A^{\prime 2} /\left(4 A^{2} B\right)-A^{\prime} B^{\prime} /\left(4 A B^{2}\right) \\
+A^{\prime} C^{\prime} /(4 A B C)=f_{E}
\end{array} \\
\\
\left(\begin{array}{c}
\text { A.6) }-8 \pi T_{4}^{4}=C^{\prime \prime} /(B C)-1 / C-B^{\prime} C^{\prime} /\left(2 B^{2} C\right)-C^{\prime 2} /\left(4 B C^{2}\right) \\
=
\end{array}\right.
\end{gathered}
$$

where

$$
f_{E} \equiv 4 \pi K^{2} / C^{2}
$$

Subtracting equation (A.6) from equation (A.4) leads, as in the uncharged case, to

$$
C^{\prime 2}=J A B C
$$

where $J$ is an integration constant. Substituting this into equation (A.4) gives

$$
A^{\prime}+\frac{C^{\prime}}{2 C} A=\left(\frac{1}{C}-\frac{4 \pi K^{2}}{C^{2}}\right) \frac{2 C^{\prime}}{J}
$$

which integrates at once to

$$
A=4 / J-\alpha / \sqrt{C}+16 \pi K^{2} /(J C)
$$

with $\alpha$ being the constant of integration. Finally, using equation (A.8) to eliminate $B$ from equation (A.5) and eliminating $A$ from the result via equation (A.10) shows, after some algebra, that equation (A.4) is satisfied identically. Thus, equations (A.8) and (A.10) constitute the general solution of equations (A.4)-(A.7).

Now, application of postulate (vii) to equation (A.1) shows that $C$ must behave like $r^{2}$ as $r \rightarrow \infty$. Hence, from equation (A.10), $A \rightarrow 4 / J$ as $r \rightarrow \infty$ and thus, by (vii) again, $J=4$. Moreover, substituting equation (A.8) into the RHS of equation (A.2) and letting $r \rightarrow \infty$ gives

$$
\phi^{\prime} \sim-K / r^{2} \text { as } r \rightarrow \infty
$$

whence

$$
K=q /(4 \pi)^{1 / 2}
$$

where $q$ denotes the charge on the particle in relativistic esu $(c=G=1)$.

Collecting these results, we have

$$
\begin{gathered}
A=1-2 m / \sqrt{C}+q^{2} / C \\
B=C^{\prime 2} /(4 A C)
\end{gathered}
$$

where $\alpha$ has been identified with twice the charge's field-producing mass by comparison with the Newtonian potential for large $r$. From equations (A.14) and (A.4) we see that $C^{\prime}$ cannot be zero for $r>0$, so that it must always be positive or always be negative. Of these two possibilities the latter is ruled out by the requirement that $C \sim r^{2}$ for large $r$. Consequently we conclude: 
- $C$ is any positive, analytic, strictly monotonic increasing function of $r$ that behaves like $r^{2}$ as $r \rightarrow \infty$.

The only other constraints on $C$ that are a consequence of the historical postulates are those induced by the necessity of satisfying equation (15) and (viii). These matters are dealt with in Appendix B.

\section{Appendix B. ADDITIONAL CONSTRAINTS ON $C$ DUE TO THE HISTORICAL POSTULATES}

As shown in Appendix A, $C$ must be positive and strictly monotonicincreasing. Hence by equation (A.14), $B$ will be positive iff $A$ is. Thus the satisfaction of condition (15) requires only that $C$ be chosen so as to make $A>0$. Rewriting equation A.13) in the form

$$
A=\frac{(\sqrt{C}-m)^{2}+q^{2}-m^{2}}{C}
$$

we see that in order to make the numerator of equation (B.1) positive and thus comply with condition (15), it is necessary that either

$$
\sqrt{C}>m+\left(m^{2}-q^{2}\right)^{1 / 2} \equiv r_{+}^{*}
$$

or

$$
\sqrt{C}<m-\left(m^{2}-q^{2}\right)^{1 / 2} \equiv r_{-}^{*}
$$

But, since it was shown in Appendix A that $C$ must behave like $r^{2}$ as $r \rightarrow \infty$, it follows that $C$ cannot satisfy equation (B.3) for all $r>0$. Consequently, the satisfaction of (15) imposes the additional requirement of inequality (B.2) on $C$. Finally, inspection of equation (B.1) shows that for $C$ constrained by inequality (B.2), the Kretschmann scalar $f=R_{i j k m} R^{i j k m}$ is well behaved as $r \rightarrow 0$, and thus (since the spherical symmetry ensures that all other scalars are necessarily functions of $f$ ) there is no curvature-related singularity there.

\section{Appendix C. EVAluation of $f$}

The nonvanishing components of $R_{i j k m}$ for equations (11)-(3) are readily found to be

$$
\begin{array}{r}
R_{1212}=\left(2 B C C^{\prime \prime}-B^{\prime} C C^{\prime}-B C^{\prime 2}\right) /(4 B C) \\
R_{1313}=R_{1212} \sin ^{2} \theta \\
R_{1414}=\left(B A^{\prime 2}+A A^{\prime} B^{\prime}-2 A B A^{\prime \prime}\right) /(4 A B) \\
R_{2323}=\left(C^{\prime 2}-4 B C\right) \sin ^{2} \theta /(4 B) \\
R_{2424}=-A^{\prime} C^{\prime} /(4 B) \\
R_{3434}=R_{2424} \sin ^{2} \theta
\end{array}
$$

together with their counterparts obtained by interchange of the indices in the first pair or the second pair.

The corresponding values of $R^{i j k m}$ are 


$$
\begin{array}{lr}
\text { (C.7) } & R^{1212}=R_{1212} /\left(B^{2} C^{2}\right) \\
\text { (C.8) } & R^{1313}=R_{1313} /\left(B^{2} C^{2} \sin ^{4} \theta\right) \\
\text { (C.9) } & R^{1414}=R_{1414} /\left(A^{2} B^{2}\right) \\
\text { (C.10) } & R^{2323}=R_{2323} /\left(C^{4} \sin ^{4} \theta\right) \\
\text { (C.11) } & R^{2424}=R_{2424} /\left(A^{2} C^{2}\right) \\
\text { (C.12) } & R^{3434}=R_{3434} /\left(A^{2} C^{2} \sin ^{4} \theta\right)
\end{array}
$$$$
\text { (C.9) } \quad R^{1414}=R_{1414} /\left(A^{2} B^{2}\right)
$$$$
\text { (C.11) } \quad R^{2424}=R_{2424} /\left(A^{2} C^{2}\right)
$$

and their associated counterparts.

Taking account of the multiplicity of terms obtainable by interchanging indices, it readily follows that

$$
\begin{array}{r}
f=4\left(R_{1212} R^{1212}+R_{1313} R^{1313}+R_{1414} R^{1414}\right. \\
+R_{2323} R^{2323}+R_{2424} R^{2424}+R_{3434} R^{3434} \\
=f_{1}+f_{2}+f_{3}+f_{4}
\end{array}
$$

where

$$
\begin{array}{r}
f_{1} \equiv 2\left[C^{\prime \prime}-B^{\prime} C^{\prime} /(2 B)-C^{\prime 2} /(2 C)\right]^{2} /\left(B^{2} C^{2}\right) \\
f_{2} \equiv\left[A^{\prime 2} /(2 A)+\left(A^{\prime} B^{\prime}\right) / 2 B-A^{\prime \prime}\right]^{2} /\left(A^{2} B^{2}\right) \\
f_{3} \equiv 4\left[C^{\prime 2} /(4 B)-C\right]^{2} / C^{4} \\
f_{4} \equiv A^{\prime 2} C^{\prime 2} /\left(2 A^{2} B^{2} C^{2}\right)
\end{array}
$$

Elimination of $B$ via equation (A.14) reduces these to

$$
\begin{array}{r}
f_{1}=8{A^{\prime}}^{2} / C^{\prime 2} \\
f_{2}=16{A^{\prime}}^{2} C^{2}\left[A^{\prime \prime} / A^{\prime}-C^{\prime \prime} / C^{\prime}+C^{\prime} /(2 C)\right]^{2} / C^{4} \\
f_{3}=4(A-1)^{2} / C^{2}
\end{array}
$$

$$
f_{4}=f_{1}
$$

Finally, elimination of $A$ via equation (A.13) gives

$$
\begin{gathered}
f_{1}=f_{4}=8\left(m / C^{3 / 2}-q^{2} / C^{2}\right)^{2} \\
f_{2}=4\left(3 q^{2} / C^{2}-2 m / C^{3 / 2}\right)^{2} \\
f_{3}=4\left(q^{2} / C-2 m / \sqrt{C}\right)^{2} / C^{2}
\end{gathered}
$$

which upon substitution into equation (C.14) give

$$
f=8\left[6\left(m \sqrt{C}-q^{2}\right)^{2}+q^{4}\right] / C^{4}
$$

\section{Appendix D. EVALUATION OF $a_{0}$}

As shown in Doughty (1981) [24], the acceleration of a neutral test particle relative to a fixed observer in a universe whose metric can be written in the 
form of equation (14) is given by

$$
a=\frac{\sqrt{-g_{r r}}\left(-g^{r r}\right)\left|g_{t t, r}\right|}{2 g_{t t}} \equiv \frac{\left|A^{\prime}\right|}{2 A \sqrt{B}}
$$

Using equation (A.13) to obtain $A^{\prime}$ and equation (A.14) to eliminate $B$, this gives

$$
a=\frac{\left|m \sqrt{C}-q^{2}\right|}{C \sqrt{A C}}
$$

As $r \rightarrow 0$, this approaches

$$
a_{0}=\frac{\left|b m-q^{2}\right|}{b^{2} \sqrt{b^{2}-2 b m+q^{2}}}
$$

\section{ACKNOWLEDGMENTS}

It is a pleasure to acknowledge several exchanges of correspondence with G.F.R. Ellis and R. Geroch, as well as numerous conversations with B. O'Neill and R. Greene. Such errors as remain are, of course, mine alone. 


\section{REFERENCES}

[1] Tipler, F.J., Clarke, C.J.S., and Ellis, G.F.R., General Relativity and Gravitation: One Hundred Years After the Birth of Albert Einstein, A. Held, ed., Plenum Press, New York, (1980) p. 97.

[2] Hawking, S. and Ellis, G.F.R., The Large Scale Structure of Space-Time, Cambridge University Press, Cambridge, (1973) p. 276.

[3] Geroch, R., J. Math. Phys. 9 (1968) 450.

[4] Sachs, R. and Wu, H., General Relativity for Mathematicians, Springer-Verlag, New York. (1977).

[5] Carter, B., Phys. Lett. 21 (1963) 423.

[6] Graves, J.C. and Brill, D., Phys. Rev. 120 (1960) 1507.

[7] Fronsdal, C., Phys. Rev. 116 (1959) 778.

[8] Kruskal, M.D., Phys. Rev. 119 (1960) 1743.

[9] Abrams, L.S., Can. J. Phys. 67 (1989) 919. (E). 74 (1996) 701.

[10] Nordström, G., K. Nederlandse Akad. van Wetenschappen, Proceedings 20 (1918) 1238.

[11] Reissner, H., Ann. d. Phys. 50 (1916) 106.

[12] Pekeris, C.L., Proc. Nat. Ac. Sci. USA 79 (1982) 6404.

[13] Hönl, H. and Papapetrou, A., Zeitsch. f. Phys. 112 (1939) 65.

[14] Schwarzschild, K., S.B. Preuss. Akad. zu Berlin I (1916) 189.

[15] O'Neill, B., Semi-Riemannian Geometry, Academic Press, New York (1983).

[16] Eiesland, J., Trans. Am. Math. Soc. 27 (1925) 213.

[17] Stavroulakis, N., Ann. Fond. L. de Broglie 6 (1981) 287.

[18] Oliver, M.A., Gen. Rel. Grav. 8 (1977) 963.

[19] Ellis, G.F.R., and Schmidt, B., Gen. Rel. Grav. 8 (1977) 915.

[20] Abrams, L.S. Physica A 227 (1996) 131.

[21] Lake, K., and Roeder, R.C., Phys. Rev. D15 (1977) 3513.

[22] Janis, A.I., Newman, E.T., and Winicour, J., Phys. Rev. Lett. 20 (1968) 878.

[23] Tolman, R.C., Relativity, Thermodynamics and Cosmology, Oxford University Press, Oxford (1934).

[24] Doughty, N., Am. J. Phys. 49 (1981) 412.

24345 Crestlawn Street, Woodland Hills, California 91367

E-mail address: ag272@lafn.org 